\title{
Neuropeptide S Enhances Memory During the Consolidation Phase and Interacts with Noradrenergic Systems in the Brain
}

\author{
Naoe Okamura', Celia Garau', Dee M Duangdao',2, Stewart D Clark', Kay Jüngling ${ }^{3}$, Hans-Christian Pape ${ }^{3}$ \\ and Rainer K Reinscheid*,1,2,4 \\ 'Department of Pharmaceutical Sciences, University of California Irvine, Irvine, CA, USA; ${ }^{2}$ Department of Molecular Biology and Biochemistry, \\ University of California Irvine, Irvine, CA, USA; ${ }^{3}$ Institute of Physiology I, Westfälische Wilhelms-University Münster, Münster, Germany; \\ ${ }^{4}$ Department of Pharmacology, University of California Irvine, Irvine, CA, USA
}

Neuropeptide S (NPS) has been shown to promote arousal and anxiolytic-like effects, as well as facilitation of fear extinction. In rodents, NPS receptors (NPSR) are prominently expressed in brain structures involved in learning and memory. Here, we investigate whether exogenous or endogenous NPS signaling can modulate acquisition, consolidation, or recall of emotional, spatial, and contextual memory traces, using two common behavioral paradigms, inhibitory avoidance (IA) and novel object recognition. In the IA paradigm, immediate and delayed post-training central NPS administration dose dependently enhanced memory retention in mice, indicating that NPS may act during the consolidation phase to enhance long-term memory. In contrast, pre-training or pre-test NPS injections were ineffective, suggesting that NPS had no effect on IA memory acquisition or recall. Peripheral administration of a synthetic NPSR antagonist attenuated NPS-induced IA memory enhancement, showing pharmacological specificity. NPS also enhanced hippocampal-dependent non-aversive memory in the novel object recognition task. In contrast, NPSR knockout mice displayed deficits in IA memory, novel object recognition, and novel place or context recognition, suggesting that activity of the endogenous NPS system is required for memory formation. Blockade of adrenergic signaling by propranolol attenuated NPS-induced memory enhancement in the IA task, indicating involvement of central noradrenergic systems. These results provide evidence for a facilitatory role of NPS in long-term memory, independent of memory content, possibly by acting as a salience signal or as an arousal-promoting factor.

Neuropsychopharmacology (20II) 36, 744-752; doi:I 0.1038/npp.2010.207; published online 8 December 2010

Keywords: neuropeptide; memory; consolidation; inhibitory avoidance; object recognition; mice

\section{INTRODUCTION}

Learning and memory can be divided into three functionally distinct phases, that is, acquisition, consolidation, and retrieval, each of which is differentially sensitive to behavioral and pharmacological interventions. Memory consolidation occurs during the first few hours following acquisition and is characterized by progressive strengthening of the memory trace (McGaugh, 1966, 2000). The inhibitory avoidance (IA) paradigm has been used extensively to study mechanisms of aversive learning and memory and the effects of drugs to manipulate these mechanisms (McGaugh and Roozendaal, 2009). A common model for non-aversive declarative memory is the object recognition task. For both

*Correspondence: Dr RK Reinscheid, Department of Pharmaceutical Sciences, University of California Irvine, 2214 Natural Sciences I, Irvine, CA 92697-3958, USA, Tel: + I 949824 9228, Fax: + I 9498242949 , E-mail: rreinsch@uci.edu

Received 7 August 2010; revised 4 October 2010; accepted 21 October 2010 paradigms, extensive studies have identified the hippocampus and amygdala as critical substrates for consolidation of long-term memories. In addition, activation of central noradrenergic signaling and peripheral stress hormones was found to enhance memory consolidation (McGaugh and Roozendaal, 2009; Dornelles et al, 2007). However, the precise neurochemical mechanisms promoting memory consolidation are still incompletely understood. As aberrant processing of memories can contribute significantly to psychopathological conditions such as post-traumatic stress disorder or anxiety disorders, it is obligatory to identify endogenous signaling pathways that contribute to this important cognitive process.

Neuropeptide S (NPS) is a recently identified peptide transmitter in the brain ( $\mathrm{Xu}$ et al, 2004) that shows remarkable conservation among tetrapod vertebrates (Reinscheid, 2007). NPS and its cognate receptor, NPSR, modulate multiple brain functions, including sleep, arousal, and emotional processing (Xu et al, 2004; Okamura and Reinscheid, 2007; Rizzi et al, 2008; Leonard et al, 2008). Conversely, NPSR knockout (KO) mice display increased 
anxiety-like behaviors and attenuated arousal (Duangdao et $a l, 2009)$. In rodents, NPS precursor transcripts are expressed in only a few brainstem structures, especially in a neuronal cluster adjacent to the noradrenergic locus coeruleus that has a key role in attention, arousal, stress, and memory (Aston-Jones, 2005). In contrast, widespread NPSR expression is detected in the brain, including the amygdaloid complex, subiculum, and cortex (Xu et al, 2004, 2007). Although the amygdala has a central role in emotional memory processing (McGaugh, 2002; Phelps and LeDoux, 2005), the subiculum is believed to filter and/ or amplify memory-related neuronal events between the hippocampus and cortex (Deadwyler and Hampson, 2004; O’Mara, 2006).

Two recent studies suggested a role for NPS in modulating learning and memory. First, local NPS administration into the endopiriform nucleus attenuated expression of contextual fear (Meis et al, 2008). Second, we showed that intra-amygdala NPS administration did not affect acquisition of cued conditioned fear, but facilitated fear extinction (Jüngling et al, 2008). Furthermore, a selective smallmolecule NPSR antagonist (SHA 68) attenuated fear extinction, indicating activity of the endogenous NPS system during extinction of conditioned fear. Both studies also found evidence for NPS-mediated enhancement of glutamatergic neurotransmission in amygdala circuits that might explain these behavioral effects.

On the basis of these previous studies and the prominent expression of NPSR transcripts in learning- and memoryassociated brain areas, we investigated the role of NPS in aversive and non-aversive memory paradigms, which are associated with amygdaloidal and hippocampal processing, respectively. Pharmacological studies were complemented by phenotypical analysis of NPSR KO mice to assess contributions of endogenous NPS signaling. Furthermore, we examined functional interactions of the NPS system with adrenergic neurotransmission, in view of the modulatory role of noradrenergic systems in memory consolidation.

\section{MATERIALS AND METHODS}

\section{Chemicals}

NPS was synthesized by the Peptide Proteomic Centre, Brain Research Centre, University of British Columbia (Vancouver, BC, Canada) and stock solutions were dissolved in water. The NPS receptor antagonist SHA 68 was synthesized as described before (Okamura et al, 2008). Other chemicals were purchased from Sigma (St Louis, $\mathrm{MO})$.

\section{Animals and Drug Administration}

Male C57Bl/6 mice (National Cancer Institute, Bethesda, $\mathrm{MD}$ ), age 8-14 weeks, were group housed under controlled conditions (temperature $21^{\circ} \mathrm{C} ; 12 \mathrm{~h}$ light-dark cycle, lights on 0600 hours) with food and water ad libitum. Before intracerebroventricular (i.c.v.) injections, mice were briefly anesthetized with isoflurane. NPS was dissolved in phosphate-buffered saline (PBS, $\mathrm{pH} 7.4$ ), $0.1 \%$ bovine serum albumin, and injected transcranially into the lateral ventricle (total volume: $2 \mu \mathrm{l}$ ) as described (Laursen and
Belknap, 1986). Correct placement of injections was verified after the experiments by histological examination and animals with misplaced i.c.v. injections were excluded. Propranolol was dissolved in isotonic saline and injected intraperitoneally (i.p., $100 \mu \mathrm{l}$ per animal). SHA 68 was dissolved in PBS, $10 \%$ Cremophor EL (Sigma), and injected i.p. (100 $\mu$ l per animal). NPSR KO mice are in a 129S6/ SvEvTac background (Allen et al, 2006). Male littermates (8-14 weeks) of all genotypes, obtained from heterozygous breeding pairs, were used for behavioral experiments. Male NPSR KO mice were previously found to display attenuated arousal and exploratory behavior, mildly increased anxietylike behaviors, altered circadian activity, and improved performance in motor learning. No phenotypical differences were observed in growth rate, body weight, depression-like behaviors, and startle responses (Duangdao et al, 2009). All animal experiments were in compliance with the National Research Council Guidelines (2003) and were approved by the University of California Irvine Institutional Animal Care and Use Committee.

\section{Step-through IA}

Mice were trained and tested on a step-through IA apparatus as described previously (Castellano et al, 1999) (see Supplementary Information for details).

\section{Hot-Plate Analgesia}

Male NPSR KO mice and their wild-type (WT) and heterozygous (HET) littermates were tested for nociceptive thresholds in the hot-plate test as described before (Chung et al, 2006). Briefly, mice were placed on a hot-plate apparatus (Saur Laborbedarf, Reutlingen, Germany) set at $56^{\circ} \mathrm{C}$ and latencies to paw licking and initiation of escape jumping were recorded. Cutoff time was set at $60 \mathrm{~s}$ to avoid tissue damage. Each animal was tested only once for thermal pain sensitivity.

\section{Novel Object Recognition}

Novel object recognition was measured similar to a previously published protocol (Tang et al, 1999); however, single-housed mice were tested in their home cages (see Supplementary Information for details).

\section{Statistical Analysis}

Data were processed by one- or two-way analysis of variance (ANOVA), wherever appropriate. Two-way ANOVA was carried out with genotype $\times$ time or treatment $\times$ time as variables, respectively, followed by twotailed post hoc tests, wherever appropriate. Novel object recognition data were analyzed by one-sample $t$-tests to examine whether object exploration times were different from the $50 \%$ chance level. Discrimination indices for each session and combination of objects were calculated from the formula (time spent with object $\mathrm{A}$-time spent with object B)/(total time exploring both objects). $P$-values $<0.05$ were considered significant. Calculations were performed with Prism 4 (GraphPad Software, San Diego, CA) or SPSS 12.0J (SPSS, Chicago, IL). Data are presented as means \pm SEM. 

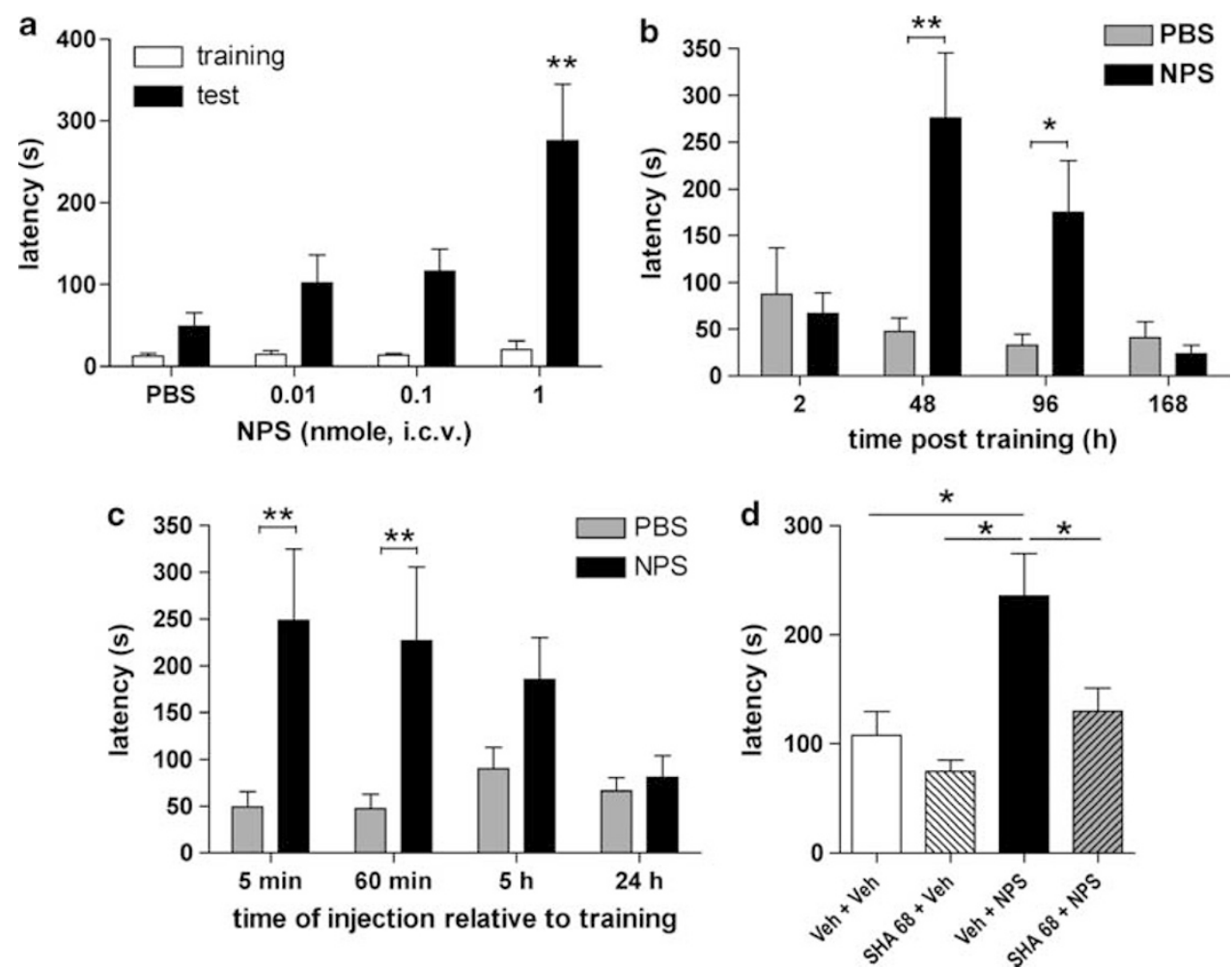

Figure I Dose- and time-dependent enhancement of inhibitory avoidance (IA) memory by neuropeptide S (NPS). (a) Male C57BI/6 mice were injected centrally with either vehicle or increasing doses of NPS 5 min after training and IA latencies were tested $48 \mathrm{~h}$ later. Phosphate-buffered saline (PBS), $n=14$; NPS 0.0 I $(n=12), 0.1(n=14)$, and I nmol $(n=12)$; **⿻丷木 $p<0.0$ I vs PBS group after one-way analysis of variance (ANOVA) with Dunnett's post hoc test. (b) Time course of IA latencies in animals injected with vehicle or I nmol NPS (intracerebroventricularly (i.c.v.)) 5 min after training and tested for memory retention at the indicated times. Separate groups of mice were used for each time interval. Animal numbers tested at 2, 48, 96, or I68 h, respectively: PBS, $n=6,16,8,9 ;$ NPS, $n=9,12,8,9$. *** $p<0.01$, two-way ANOVA with Bonferroni's post hoc test. (c) Influence of administration time on NPS-induced memory enhancement in the IA paradigm. Male C57BI/6 mice were injected centrally with I nmol NPS or vehicle at the indicated delays after training and IA latencies were measured $48 \mathrm{~h}$ later. Animal numbers injected at $5 \mathrm{~min}, 60 \mathrm{~min}, 5$ or $24 \mathrm{~h}$ relative to training: vehicle $(8,14,12,1 \mathrm{I}$, and I I) and NPS (8, I0, 9, I2, and 9). *** $<0.01$, *p $<0.05$, two-way ANOVA with Bonferroni's post hoc test. (d) Blockade of NPS-induced memory enhancement by the NPSR antagonist 3-oxo-I,I-diphenyl-tetrahydro-oxazolo[3,4-a]pyrazine-7-carboxylic acid 4-fluoro-benzylamide (SHA 68). Naive mice were trained in the IA paradigm and then injected with SHA $68(50 \mathrm{mg} / \mathrm{kg}$, i.p.) or vehicle (Veh, PBS containing I0\% Cremophor EL) immediately after training, followed by central NPS (I nmol) or vehicle (Veh) administration 15 min later. IA latencies were measured $48 \mathrm{~h}$ later. Veh + Veh, $n=9 ; \mathrm{SHA} 68+\mathrm{Veh}, n=9 ; \mathrm{Veh}+\mathrm{NPS}$, $n=25 ;$ SHA $68+$ NPS, $n=26$. * $p<0.05$ one-way ANOVA with Bonferroni's post hoc test.

\section{RESULTS}

\section{Step-Through IA Test}

Across several series of IA studies, average entrance latencies during training sessions, that is, before shock exposure, were $14.07 \pm 3.3 \mathrm{~s}$. One-way ANOVA for preshock latencies between treatment groups in all studies detected no difference, indicating that all animals readily entered the shock compartment upon first exposure. In all experiments, vehicle-treated mice showed statistically longer test latencies compared with their training latencies, indicating retained memory of the mild foot shock. However, the relatively mild intensity of the foot shock $(0.2 \mathrm{~mA}, 1 \mathrm{~s})$ was not sufficient to induce saturating effects, thus allowing for detection of enhanced memory retention.

Immediate post-training i.c.v. injections of NPS produced enhanced retention of the aversive memory in a dosedependent manner, compared with PBS-injected animals, when mice were tested $48 \mathrm{~h}$ after training (Figure 1a). Oneway ANOVA showed significant differences between treatment groups $\left(\mathrm{F}_{3,48}=5.94, p=0.0016\right)$, and post hoc tests revealed a significant increase in step-through latency between vehicle and $1 \mathrm{nmol}$ NPS-treated animals $(p<0.01)$.

Next, we examined the time course and duration of NPSinduced memory enhancement. Following training and injection of $1 \mathrm{nmol}$ NPS or PBS, IA latencies were measured after $2,48,96$, or $168 \mathrm{~h}$, respectively (Figure $1 \mathrm{~b}$ ). Two-way ANOVA showed significant differences in latencies depending on treatment and time, indicating that NPS treatment produced transient memory enhancement peaking at $48 \mathrm{~h}$, but declining thereafter (treatment: $\mathrm{F}_{1,69}=8.52, p=0.0047$; time: $\mathrm{F}_{3,69}=4.47, p=0.0063$; interaction: $\mathrm{F}_{3,69}=5.38$, $p=0.0022)$. Post hoc tests revealed significant differences between the two treatment groups at 48 and $96 \mathrm{~h}$ after training, but not at 2 or $168 \mathrm{~h}$. These observations suggest that NPS may positively modulate long-term memory for at least 4 days after training, but has no effect on short-term memory. However, NPS-induced long-term memory enhancement cannot be sustained for 7 days when latencies approach levels seen in vehicle-treated animals. It should be noted that in both treatment groups, IA memory retention latencies at 7 days post-training were still significantly higher than their own training latencies (time/training: 
$\mathrm{F}_{1,32}=5.13, \quad p=0.0304 ; \quad$ drug treatment: $\mathrm{F}_{1,32}=0.55$, $p=0.4623$; interaction: $\mathrm{F}_{1,32}=0.89, p=0.353$ ), suggesting successful consolidation of moderate strength IA memory as a result of training, but not drug treatment.

Pharmacological manipulations of long-term memory are strongly dependent on the time of drug administration relative to the time of training (McGaugh and Roozendaal, 2009). Therefore, we injected NPS or vehicle at various time points ( $5 \mathrm{~min}, 1,5$, or $24 \mathrm{~h}$ after training, respectively) and compared 48-h retention (Figure 1c). Two-way ANOVA showed significant differences in latencies to enter the shock compartment as a result of treatment (treatment: $\mathrm{F}_{1,80}=18.09, \quad p<0.0001 ;$ time: $\mathrm{F}_{3,80}=1.35, \quad p=0.2644$; interaction: $F_{3,80}=2.12, p=0.1041$ ) and Bonferroni's post hoc test revealed significantly increased IA latencies when NPS was injected 5 and 60 min after training. When NPS was injected $5 \mathrm{~h}$ post-training, mean latencies were still higher than that in vehicle-treated animals, but failed to reach significance $\left(t_{21}=1.831, p=0.08\right.$; unpaired $t$-test for test latencies after 5 - $h$ delay of injection). Delayed administration of NPS $24 \mathrm{~h}$ after training had no effect on IA memory retention. The observation that both immediate and delayed infusions of NPS are able to enhance long-term memories indicates that NPS might act during the consolidation phase by influencing long-lasting neuronal plasticity.

The next set of experiments examined whether NPS might influence acquisition or recall of IA memory. Pre-training NPS injections of $1 \mathrm{nmol}$ NPS or vehicle produced increased IA latencies in both groups of animals compared with their training latencies (training: $\mathrm{F}_{1,34}=9.792, p=0.0036$; twoway ANOVA); however, NPS-treated animals showed only moderately increased latencies (Supplementary Figure S1A). No statistically significant difference in IA latencies was observed between vehicle- and NPS-treated groups injected before training $\left(\mathrm{F}_{1,34}=1.347, p=0.25\right)$ and two-way ANO$\mathrm{VA}$ indicated no interaction between time and treatment $\left(\mathrm{F}_{1,34}=1.522, p=0.22\right)$. When $1 \mathrm{nmol}$ NPS or vehicle were injected $15 \mathrm{~min}$ before the recall session, significant increases in recall latencies $v s$ training latencies were observed in both groups of mice. Two-way ANOVA indicated significant effects of time (ie, training $v s$ test; $\left.\mathrm{F}_{1,34}=20.86, \quad p<0.0001\right)$ and treatment $\left(\mathrm{F}_{1,34}=5.48\right.$, $p=0.0253)$ with positive interaction $\left(\mathrm{F}_{1,34}=5.29\right.$, $p=0.0277)$. Pre-retrieval administration of NPS reduced the average latency to enter the shock compartment, as recall latencies in NPS-injected mice were significantly lower than in vehicle-treated animals $(p<0.01$; Bonferroni post hoc test) (Supplementary Figure S1B). The results from pre-training or pre-retrieval NPS administrations might suggest an acute amnesic effect of NPS, but are more likely caused by the acute anxiolytic properties of the peptide.

To test the specificity of NPS-induced memory enhancement, we co-administered the selective NPSR antagonist SHA 68. SHA $68(50 \mathrm{mg} / \mathrm{kg}$, i.p.) or vehicle were injected immediately after training, followed by central administration of $1 \mathrm{nmol}$ NPS or vehicle $15 \mathrm{~min}$ later, and IA latencies were tested after $48 \mathrm{~h}$. One-way ANOVA revealed that only mice treated with vehicle + NPS displayed significantly increased latencies when compared with vehicle + vehicle-treated animals $\left(\mathrm{F}_{3,67}=4.755, p=0.0046\right.$; Figure 1d). Co-administration of SHA 68 blocked NPS-

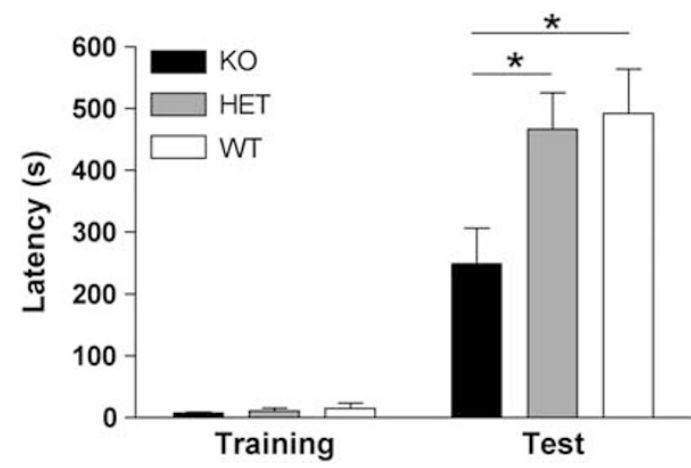

Figure 2 Long-term memory deficits in neuropeptide $\mathrm{S}$ receptor (NPSR) knockout (KO) mice. NPSR KO mice display attenuated 48-h retention latencies in the inhibitory avoidance (IA) paradigm compared with their heterozygous (HET) and wild-type (WT) littermates. $\mathrm{KO}, n=19$; HET, $n=14$; WT, $n=10$. * $p<0.05 \mathrm{KO}$ vs WT or HET, one-way analysis of variance (ANOVA) with Bonferroni's post hoc test.

induced enhancement of memory for the aversive compartment, as latencies in SHA $68+$ NPS-treated mice were significantly different from vehicle + NPS-treated animals ( $p<0.05$, one-way ANOVA with Bonferroni's post hoc test). Administration of SHA 68 alone did not produce effects different from vehicle treatment. These data indicate that the memory-enhancing effects observed after NPS administration are mediated by the activation of NPS receptors. However, treatment with SHA 68 alone did not prevent the formation of IA memory, suggesting that contribution of endogenous NPS signaling might be either small or occur at different time points.

The role of endogenous NPS signaling in learning and memory was investigated using NPSR KO mice and their heterozygous (HET) or WT littermates. As shown in Figure 2, training latencies were indistinguishable across the three genotypes. Mice of all genotypes showed significantly increased IA latencies compared with their training latencies, indicating that associations of the aversive stimulus and the context had been formed. However, one-way ANOVA indicated that NPSR KO mice displayed significantly reduced latencies to enter the shock compartment $48 \mathrm{~h}$ after training when compared with their WT or HET littermates $\left(F_{2,41}=5.009, p=0.0113\right)$, and Bonferroni post hoc tests showed that IA latencies of NPSR KO mice are significantly different from both WT and HET littermates $(p<0.05)$. These results indicate that endogenous NPS signaling might modulate the strength of long-term memory or that NPS is required for prolonged behavioral arousal that might facilitate memory recall. Absolute IA latencies of NPSR WT and HET mice (129S6/SvEvTac background) were significantly higher than in NPS- or vehicle-injected C57Bl/6 mice. Superior performance of $129-S v E v$ mice in learning and memory paradigms has been reported previously and our data confirm this observation (Crawley et al, 1997). As experiments involving foot-shock delivery can be confounded by the nociceptive status of the animals, pain sensitivity was tested in NPSR KO and WT mice. However, no difference in nociceptive thresholds between the genotypes was detected in the hot-plate test (Supplementary Figure S2). 


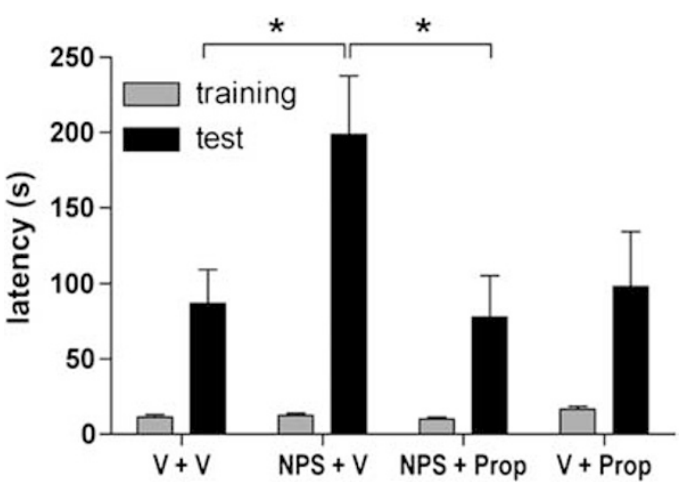

Figure 3 Propranolol blocks neuropeptide S (NPS)-induced memory enhancement. Mice were trained in the inhibitory avoidance (IA) paradigm and injected with propranolol (Prop, $2 \mathrm{mg} / \mathrm{kg}$, i.p.) or vehicle (V) immediately after the training, followed by central NPS (I nmol) or vehicle administration $15 \mathrm{~min}$ later. IA latencies were measured $48 \mathrm{~h}$ after training. Animal numbers and treatment groups: $V+V, n=14$; NPS $+V, n=17$; NPS + Prop, $n=20 ; V+$ Prop, $n=20$. $* p<0.05$, one-way analysis of variance (ANOVA) followed by Bonferroni's post hoc test.

Activation of central beta-adrenergic receptors can enhance memory consolidation in the IA paradigm (Izquierdo and Dias, 1983; Introini-Collison et al, 1992; Quirarte et al, 1997), whereas blockade of central adrenergic signaling can prevent consolidation of aversive memory in animal models (Introini-Collison et al, 1994; Nielson et al, 1999) and humans (Grillon et al, 2004). Therefore, the centrally acting beta-adrenergic receptor antagonist propranolol $(2 \mathrm{mg} / \mathrm{kg} / 100 \mu \mathrm{l}$, i.p.) was used to investigate interactions between NPS and adrenergic systems, using a dose of propranolol that was previously found to effectively block memory-enhancing effects of adrenergic agonists or GABAergic antagonists, without being effective by itself (Introini-Collison et al, 1994). After training, mice received i.p. injections of either vehicle or propranolol, followed by central administration of either vehicle or $1 \mathrm{nmol}$ NPS 15 min later. One-way ANOVA revealed significant differences in 48-h IA latencies between treatment groups $\left(\mathrm{F}_{3,67}=3.564 ; p=0.0186\right)$ and Bonferroni's post hoc test showed that NPS + vehicle treatment increased IA latencies significantly compared with vehicle + vehicle- or NPS + propranolol-treated animals. Co-administration of propranolol together with NPS was able to completely block the memory-enhancing effect of NPS, whereas administration of propranolol alone was ineffective compared with vehicle controls, as had been reported previously (Introini-Collison et al, 1994) (Figure 3). These data provide evidence for an involvement of adrenergic systems in mediating NPSinduced enhancement of aversive memory.

\section{Novel Object Recognition}

The IA paradigm measures aversive contextual memory involving a highly arousing experience, that is, foot shock (Izquierdo et al, 1997). To investigate if the pro-cognitive effect of NPS is limited to emotionally aversive memories, we tested the effect of central NPS administration or genetically induced absence of NPSR signaling in a paradigm of neutral memory content, that is, the novel object recognition task. Previous studies have suggested a

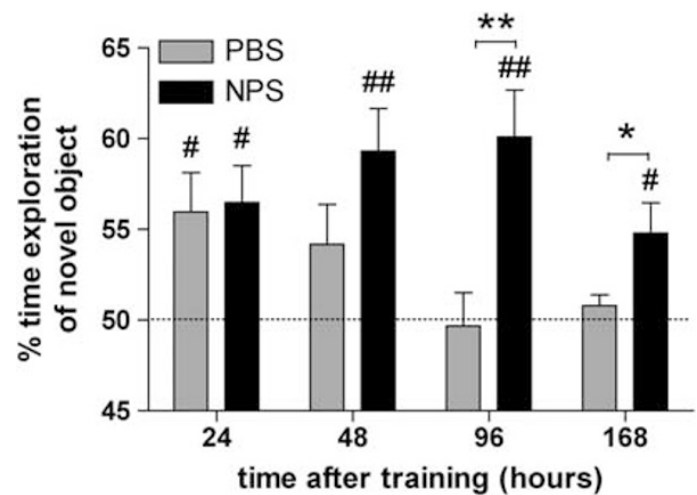

Figure 4 Neuropeptide S (NPS) enhances novel object recognition. Mice were trained with objects $A$ and $B$ for 5 min and then injected with vehicle or I nmol NPS 5 min after training. After 24, 48, 96, or $168 \mathrm{~h}$, mice explored one familiar and one novel object. NPS-injected mice spent more time exploring the novel object at longer test delays. Separate groups of mice were used for each time point. Numbers of animals at 24, 48, 96, and $168 \mathrm{~h}$ delays: phosphate-buffered saline (PBS), $n=7,7,8$, and 8 , respectively; NPS, $n=7,8,8$, and 8 , respectively. $* * * 00.01$, * $p<0.05$, two-way analysis of variance (ANOVA) with Bonferroni's post hoc test. ${ }^{\#} p<0.05$, ${ }^{\# \#} p<0.01$, one-sample t-test compared with $50 \%$ chance level. The dashed line indicates $50 \%$ exploration time if none of the objects is preferred.

significant role of the hippocampus in object recognition (Clark et al, 2000), although other studies support a regionally specific function of particular hippocampal structures (Broadbent et al, 2004) or question the role of the hippocampus for object recognition memory (Winters et al, 2004). In addition, amygdaloidal and cortical structures might also be involved (Moses et al, 2005). In pilot studies, C57Bl/6 mice displayed no preferences when various sets of two different objects were presented. However, 129S6/SvEvTac mice revealed consistent preference for one over the other object, regardless of combination. Therefore, the paradigm was modified for 129S6/ SvEvTac mice by training mice with sets of two identical objects and testing discrimination memory with one familiar and one novel object as described (see Supplementary Information for details).

C57Bl/6 mice explored both objects $\mathrm{A}$ and $\mathrm{B}$ equally during training (Supplementary Figure S3) and received $1 \mathrm{nmol}$ NPS or vehicle injections $5 \mathrm{~min}$ later. After $24 \mathrm{~h}$, both groups of mice spent significantly more time exploring the novel object, indicating that they had memorized the familiar object (Figure 4). One-sample $t$-tests for the 24-h delay showed that both groups displayed preference for the novel object that was significantly different from the $50 \%$ chance level (PBS: $t_{6}=2.758, p=0.0329$; NPS: $t_{6}=3.16$, $p=0.0196)$. When separate groups of $\mathrm{C} 57 \mathrm{Bl} / 6$ mice were trained and treated in the same way, but presentation of the novel object was delayed for 48,96 , or $168 \mathrm{~h}$, mice injected with NPS displayed increased exploration of the novel object that was significantly different from $50 \%$ chance levels at all time points ( $48 \mathrm{~h}: t_{7}=3.974, p=0.0054 ; 96 \mathrm{~h}$ : $t_{7}=3.907, p=0.0058 ; 168 \mathrm{~h}: t_{7}=2.874, p=0.0239$; onesample $t$-test). In contrast, vehicle-injected animals were unable to discriminate between the objects when testing was delayed for 48,96 , or $168 \mathrm{~h}$, respectively, and one-sample $t$-tests showed no significant difference from chance levels 

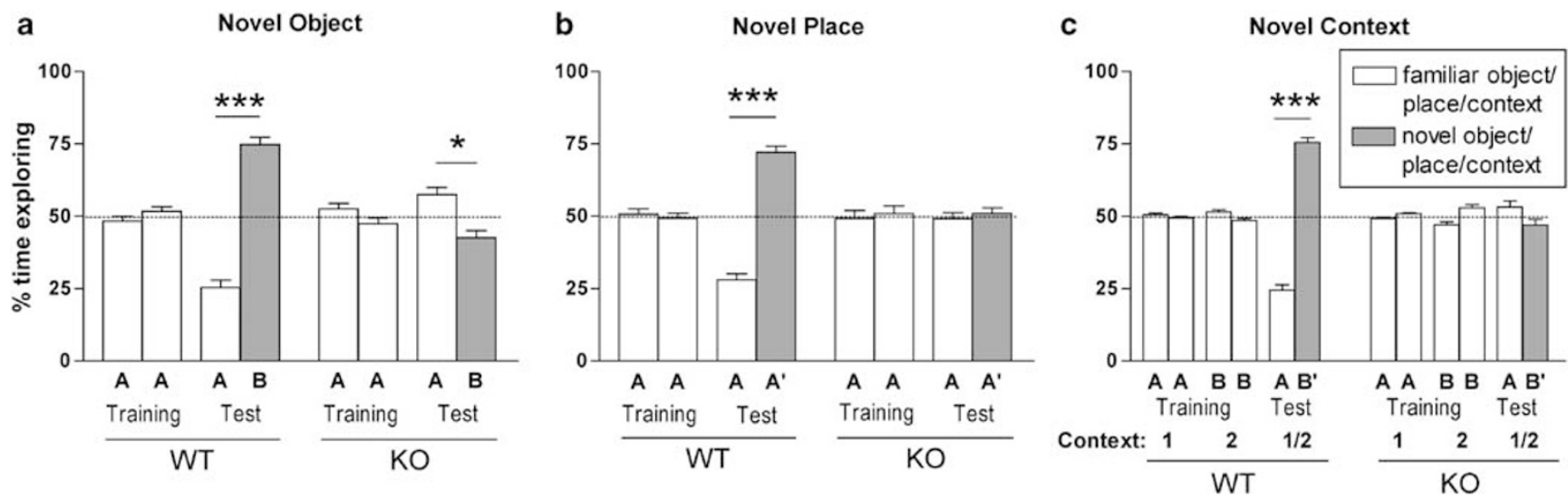

Figure 5 Impairment of object recognition memory in neuropeptide S receptor (NPSR) knockout (KO) mice. (a) Novel object recognition test. NPSR $K O, n=$ I4; NPSR wild-type (WT), $n=18$. (b) Novel place recognition test. NPSR KO = I , NPSR WT $n=1$ I. (c) Novel context recognition test. NPSR $\mathrm{KO} n=7$, NPSR WT $n=1$ I. The dashed line indicates $50 \%$ exploration time if none of the objects is preferred. $* p<0.05$, $* * * * p<0.001$; one-sample $t$-test for both familiar and novel object/place/context compared with 50\% chance level. Bars under the asterisks symbolize equal difference of both values from chance.

(48 h: $t_{6}=1.895, p=0.1068 ; 96 \mathrm{~h}: t_{7}=0.1778, p=0.8939$; $\left.168 \mathrm{~h}: t_{7}=1.341, p=0.2219\right)$. Two-way ANOVA revealed significant main effects of treatment $\left(F_{1,53}=12.46\right.$, $p=0.0009$ ) and NPS-treated mice tested 96 or $168 \mathrm{~h}$ after training showed significantly more exploratory activity toward the novel object than vehicle-treated animals ( $p<0.05$, Bonferroni post hoc test). Total object exploration time did not differ between treatment and time groups (Supplementary Table S1). The data show that object memory in vehicle-treated $\mathrm{C} 57 \mathrm{Bl} / 6$ mice is transient and can only be recalled for less than $48 \mathrm{~h}$. NPS appears to enhance long-term recognition of novel objects for up to 7 days after the training, although our data indicate that this effect might also be transient.

NPSR KO and WT mice explored the two identical objects equally during training. When tested for memory retention $24 \mathrm{~h}$ later, NPSR WT mice displayed significantly increased exploration of the novel object $v s$ the familiar one and the preference for the novel object was significantly different from $50 \%$ chance level $\left(t_{17}=9.577, p<0.0001\right.$; one-sample $t$-test), whereas NPSR KO mice showed significant novel object aversion compared with the $50 \%$ chance level $\left(t_{12}=3.014, p=0.0108\right)$ (Figure $\left.5 \mathrm{a}\right)$. This aversive reaction of NPSR KO mice might be related to novelty-induced fear, as we have previously shown that NPSR KO mice display elevated anxiety-like behaviors (Duangdao et al, 2009). Two-way ANOVA of discrimination indices (Supplementary Figure $\mathrm{S} 4 \mathrm{~A})$ revealed main effects of genotype $\left(\mathrm{F}_{1,59}=66.77\right.$, $p<0.0001)$ and training $\left(\mathrm{F}_{1,59}=16.27, p<0.0002\right)$ with positive interaction $\left(\mathrm{F}_{1,59}=39.38, p<0.0001\right)$. Post hoc Bonferroni tests showed significant object discrimination in NPSR WT $(p<0.001)$, but not KO mice $(p>0.05)$. In the novel place recognition test, similar observations were made: NPSR KO mice failed to recognize the object that had been moved to a new location $\left(t_{10}=0.4278, p=0.6778\right.$; onesample $t$-test), whereas NPSR WT mice spent significantly more time exploring the relocated object compared with chance levels $\left(t_{10}=10.34, p<0.0001\right)$ (Figure 5b). Two-way ANOVA showed significant effects of genotype $\left(\mathrm{F}_{1,40}=\right.$ 19.65, $p<0.0001)$ and training $\left(\mathrm{F}_{1,40}=26.96, p<0.0001\right)$ with positive interaction $\left(\mathrm{F}_{1,40}=26.11, p<0.0001\right)$ (Supplementary Figure S4B). Bonferroni post hoc tests confirmed significant discrimination between stationary and relocated object in NPSR WT $(p<0.001)$, but not NPSR $\mathrm{KO}$ mice $(p>0.05)$. In the novel context recognition paradigm, NPSR KO mice were unable to discriminate between two objects $\left(t_{6}=1.475, p=0.1907\right.$, one-sample $t$-test comparing to $50 \%$ chance level), of which one had been presented in a different context before, whereas NSPR WT mice showed significant exploration of the object that did not match the previously learned context $\left(t_{10}=15.00\right.$, $p<0.0001$, one-sample $t$-test) (Figure $5 \mathrm{c}$ ). Two-way ANOVA revealed significant main effects of genotype $\left(\mathrm{F}_{1,48}=55.65\right.$, $p<0.0001)$ and training $\left(\mathrm{F}_{2,48}=48.75, p<0.0001\right)$ with positive interaction $\left(F_{1,48}=104.05, p<0.0001\right)$ (Supplementary Figure S4C). Bonferroni post hoc tests showed significant discrimination between matching and non-matching objects in NPSR WT $(p<0.001)$, but not NPSR KO mice $(p>0.05)$, whereas no influence of training context was detected in both genotypes $(p>0.05)$ (Supplementary Figure S4C). Total object exploration times did not differ between genotypes across the three paradigms (Supplementary Table S1), although NPSR WT and KO mice tended to show shorter total exploration times during the retention tests as compared with their training explorations. These observations suggest that endogenous NPS signaling might be critically involved in the formation of declarative memory without emotional content, such as object recognition.

\section{DISCUSSION}

This study provides complementing evidence from both pharmacological and genetic models for a memory-enhancing function of NPS. In the two behavioral paradigms employed here, pharmacological activation of NPS signaling dose and time dependently enhances long-term memory, presumably by acting during the consolidation phase. Acquisition and retrieval of IA memory are attenuated by central NPS administration, most likely owing to its acute 
anxiolytic-like effects. Conversely, NPSR KO mice display significant deficits in long-term memory. Furthermore, the pro-cognitive effects of NPS are independent of emotional content as we observed NPS-dependent improvement of object recognition memory, whereas NPSR KO mice displayed the opposite phenotype. Taken together, these studies suggest that the NPS system may play a role during the consolidation phase to enhance memory strength. Alternatively, NPS might exert its effects by producing prolonged arousal that facilitates memory recall. The latter model, however, appears less likely because the in vivo effects of centrally administered NPS on locomotor activity subside after $\sim 90 \mathrm{~min}$ (Xu et al, 2004). In addition, we did not find evidence for such an arousal effect in the IA paradigm when mice were tested $2 \mathrm{~h}$ after training and injection, but instead IA latencies were significantly increased after 48 or $96 \mathrm{~h}$.

Post-training drug administrations in learning and memory paradigms ensure a drug-free state of the animals during both acquisition and recall, and exclude potentially confounding effects on sensory, motor, and motivational states that could be produced by pre-training or pre-test drug administration. NPS appears to selectively act during the consolidation phase to enhance memory since pretraining or pre-retrieval NPS administrations failed to enhance memory retention in the IA paradigm. On the other hand, NPS was still able to improve memory when administered $1 \mathrm{~h}$ - and possibly later - after the training, supporting earlier studies postulating a critical time window for consolidation that is sensitive to pharmacological intervention (McGaugh and Roozendaal, 2009). Once memory has been consolidated (eg, 24-h post-training), however, NPS is no longer able to modify such memory traces. Pre-training and pre-retrieval administrations of NPS resulted in shorter IA latencies than vehicle treatment, most likely owing to the acute anxiolytic effects of NPS (Xu et al, 2004; Rizzi et al, 2008; Leonard et al, 2008; Jüngling et al, 2008). Differential effects of pre-training $v s$ post-training drug administrations on IA memory have also been reported for benzodiazepines. Similar to NPS, pre-training administration of benzodiazepines attenuated acquisition of IA memory; however, immediate posttraining benzodiazepine administrations had no effect on IA memory assessed 24-48 h later (Patel et al, 1979; Jensen et al, 1979; Cahill et al, 1986). As both NPS and benzodiazepines produce acute anxiolytic effects, pretraining administrations may therefore attenuate perception of fearful events and thus impair formation of fearful memories. However, we cannot rule out the possibility of state dependency on IA memory retrieval. Furthermore, NPSR KO mice showed significantly attenuated memory retention in the IA task, suggesting endogenous NPS signaling being important for memory formation. Memoryenhancing effects of NPS were successfully blocked by the selective NPSR antagonist SHA 68. However, in contrast to our findings in NPSR KO mice, administration of SHA 68 alone did not attenuate IA latencies in normal mice. One possible interpretation of these data is that the time course of endogenous NPS release might be substantially different from the pharmacokinetic profile of SHA 68. Further studies investigating the time course of NPSR activation during individual stages of memory formation are certainly required.
As the aversive stimulus of the IA paradigm produces a state of heightened arousal, we also used emotionally neutral object recognition to evaluate NPS effects under low arousal conditions. Central administration of NPS significantly prolonged the retention of recognition memory in $\mathrm{C} 57 \mathrm{Bl} / 6$ mice. Both NPS- and vehicle-treated mice performed equally well when tested only $24 \mathrm{~h}$ after the training; however, recognition memory decreased in vehicletreated animals when training and test were separated by longer intervals. In contrast, mice receiving post-training NPS administrations displayed superior performance even 1 week after training, although exploration times after 7 days were approaching baseline levels, indicating a transient effect of NPS. Conversely, NPSR KO mice showed significant deficits in three different variations of the novel object recognition task, indicating that endogenous NPS signaling might be critically involved in the formation of such memories. Our observations suggest that endogenous NPS signaling is required for consolidation and/or recall of object recognition memory, as the three variants of the task tested object-specific, spatial and contextual memories, respectively. Activation of the NPS system appears to be necessary for transient enhancement of memory, independent of emotional quality, content, or context.

In both paradigms, the pro-cognitive effects of NPS appear to be transient, with peak effects at 2-4 days post-training, but gradually declining after that. Thus, NPS-induced enhancement of memory is not permanent, but rather produces a transient increase in strength with which the memory trace can be recalled. In these conditions, NPS might act as a salience signal in concert with other memory systems by temporarily enhancing valence of the memory trace. Data from NPSR KO mice support this hypothesis, as the magnitude of their IA latencies is obviously attenuated compared with WT and HET littermates, and the difference might indicate the absence of a salience signal. Finally, presentations of significantly less salient stimuli, such as in object recognition, appear to be insufficient for memory formation in NPSR KO mice, whereas object recognition memory can be enhanced and prolonged by over-stimulating NPS neurotransmission in normal mice. Alternatively, NPS may produce long-lasting - but transient - arousal through activation of secondary systems that could still affect memory recall after 3-4 days.

The anatomical substrates for the memory-enhancing effects of NPS are currently unknown; however, NPSR mRNA is prominently expressed in several amygdala and extended amygdala structures, as well as the subiculum, which contains the highest level of NPSR transcripts in the rodent brain (Xu et al, 2007). A recent study showed facilitation of fear extinction learning and recall after bilateral intra-amygdala administrations of NPS before extinction training (Jüngling et al, 2008). As extinction of previously conditioned fear responses requires new learning (Maren and Quirk, 2004), these findings suggest that activation of amygdaloidal NPS receptors may be required for the pro-cognitive effects of NPS. Interestingly, pre-training NPS injections were ineffective to modulate establishment of conditioned fear (Jüngling et al, 2008), supporting our present findings that NPS may not affect memory acquisition. Furthermore, NPS administration after successful extinction training did not affect extinction recall or renewal on the subsequent day, as both vehicle- or NPS- 
treated animals had been trained to the same level of extinction. These observations, together with our present data, indicate that NPS might act during the consolidation phase, rather than modulating memory once it has been consolidated. However, consolidation of conditioned fear did not increase upon post-training NPS administration in our previous study (Jüngling et al, 2008). This lack of effect could be owing to the high salience of the unconditioned stimulus producing maximal levels of consolidation, and thus no further enhancement by NPS could be achieved. Furthermore, NPS was locally applied to the amygdala in the Jüngling et al (2008) study, and mechanisms of fear memory consolidation involve ancillary synaptic networks in addition to the amygdala (Pape and Paré, 2010). Finally, different neuronal mechanisms are involved in the consolidation of conditioned fear $v s$ stepthrough IA memory. Noradrenergic neurotransmission in the amygdala may be a common neuronal mechanism to enhance consolidation of both aversive and emotionally neutral memories (Dornelles et al, 2007; Ferry et al, 1999; Gold and van Buskirk, 1975; Liang et al, 1986; Roozendaal et al, 2008). Our results that the adrenergic antagonist propranolol can block NPS-induced memory enhancement indicate that NPS may act via a similar mechanism.

In summary, our studies provide evidence for a significant role of the NPS system to facilitate consolidation of various types of memory, possibly by acting as a salience signal or as an arousal-promoting factor. As aberrant processing of memory-especially with aversive or traumatic content-is a critical factor in anxiety disorders, especially post-traumatic stress disorder, drugs targeting the NPS system might offer novel therapeutic avenues. A functional polymorphism in the human NPS receptor gene that significantly changes agonist sensitivity of the receptor (Reinscheid et al, 2005) was recently associated with panic disorder (Okamura et al, 2007). As the polymorphism occurs with high frequency in the human population, it should be possible to study effects of enhanced or attenuated NPS signaling with respect to human memory consolidation without requirement of drug administration.

\section{ACKNOWLEDGEMENTS}

We gratefully acknowledge scientific support by Drs Benno Roozendaal and Marcelo Wood (University of California, Irvine, CA), Natalie Rodrigue (Creascience, Montreal, Canada) for help with statistical analysis, and Dr Beverly $\mathrm{H}$ Koller (University of North Carolina at Chapel Hill) for making NPSR knockout mice available. This work was supported in part by a National Institute of Mental Health Grant (MH-71313; RKR), a postdoctoral fellowship from the Mitsubishi Pharma Research Foundation (NO), a training grant fellowship from the National Institute on Drug Abuse (DMD), a post-doctoral fellowship from the Canadian Institutes of Health Research (SDC), and a grant from the Deutsche Forschungsgemeinschaft (SFB-TRR58, TP A03; HCP), the Max-Planck Society, and Humboldt Foundation (Research Award 2007; HCP).

\section{DISCLOSURE}

The authors declare no conflict of interest.

\section{REFERENCES}

Allen IC, Pace AJ, Jania LA, Ledford JG, Latour AM, Snouwaert JN et al (2006). Expression and function of NPSR1/GPRA in the lung before and after induction of asthma-like disease. $\mathrm{Am} \mathrm{J}$ Physiol Lung Cell Mol Physiol 291: L1005-L1017.

Aston-Jones G (2005). Brain structures and receptors involved in alertness. Sleep Med 6(Suppl 1): S3-S7.

Broadbent NJ, Squire LR, Clark RE (2004). Spatial memory, recognition memory, and the hippocampus. Proc Natl Acad Sci USA 101: 14515-14520.

Cahill L, Brioni J, Izquierdo I (1986). Retrograde memory enhancement by diazepam: its relation to anterograde amnesia and some clinical implications. Psychopharmacology 90: $554-556$.

Castellano C, Cabib S, Puglisi-Allegra S, Gasbarri A, Sulli A, Pacitti C et al (1999). Strain-dependent involvement of D1 and D2 dopamine receptors in muscarinic cholinergic influences on memory storage. Behav Brain Res 98: 17-26.

Chung S, Pohl S, Zeng J, Civelli O, Reinscheid RK (2006). Endogenous orphanin FQ/nociceptin is involved in the development of morphine tolerance. J Pharmacol Exp Ther 318: $262-267$.

Clark RE, Zola SM, Squire LR (2000). Impaired recognition memory in rats after damage to the hippocampus. J Neurosci 20: 8853-8860.

Crawley JN, Belknap JK, Collins A, Crabbe JC, Frankel W, Henderson $\mathrm{N}$ et al (1997). Behavioral phenotypes of inbred mouse strains: implications and recommendations for molecular studies. Psychopharmacology 132: 107-124.

Deadwyler SA, Hampson RE (2004). Differential but complementary mnemonic functions of the hippocampus and subiculum. Neuron 42: 465-476.

Dornelles A, de Lima MN, Grazziotin M, Presti-Torres J, Garcia VA, Scalco FS et al (2007). Adrenergic enhancement of consolidation of object recognition memory. Neurobiol Learn Mem 88: 137-142.

Duangdao DM, Clark SD, Okamura N, Reinscheid RK (2009). Behavioral phenotyping of neuropeptide $S$ receptor knockout mice. Behav Brain Res 205: 1-9.

Ferry B, Roozendaal B, McGaugh JL (1999). Role of norepinephrine in mediating stress hormone regulation of long-term memory storage: a critical involvement of the amygdala. Biol Psychiatry 46: 1140-1152.

Gold PE, van Buskirk R (1975). Facilitation of time-dependent memory processes with posttrial epinephrine injections. Behav Biol 13: 145-153.

Grillon C, Cordova J, Morgan CA, Charney DS, Davis M (2004). Effects of the beta-blocker propranolol on cued and contextual fear conditioning in humans. Psychopharmacology 175: 342-352. Introini-Collison I, Saghafi D, Novack GD, McGaugh JL (1992). Memory-enhancing effects of post-training dipivefrin and epinephrine: involvement of peripheral and central adrenergic receptors. Brain Res 572: 81-86.

Introini-Collison IB, Castellano C, McGaugh JL (1994). Interaction of GABAergic and beta-noradrenergic drugs in the regulation of memory storage. Behav Neural Biol 61: 150-155.

Izquierdo I, Dias RD (1983). The influence of adrenergic receptor antagonists on the amnestic and antiamnestic actions of adrenaline and tyramine. Psychopharmacology 80: 181-183.

Izquierdo I, Quillfeldt JA, Zanatta MS, Quevedo J, Schaeffer E, Schmitz PK et al (1997). Sequential role of hippocampus and amygdala, entorhinal cortex and parietal cortex in formation and retrieval of memory for inhibitory avoidance in rats. Eur $J$ Neurosci 9: 786-793.

Jensen RA, Martinez JL, Vasquez BJ, McGaugh JL (1979). Benzodiazepines alter acquisition and retention of an inhibitory avoidance response in mice. Psychopharmacology 64: 125-126. 
Jüngling K, Seidenbecher T, Sosulina L, Lesting J, Sangha S, Clark $\mathrm{SD}$ et al (2008). Neuropeptide S-mediated control of fear expression and extinction: role of intercalated GABAergic neurons in the amygdala. Neuron 59: 298-310.

Laursen SE, Belknap JK (1986). Intracerebroventricular injections in mice. Some methodological refinements. J Pharmacol Methods 16: $355-357$.

Leonard SK, Dwyer JM, Sukoff Rizzo SJ, Platt B, Logue SF, Neal SJ et al (2008). Pharmacology of neuropeptide $S$ in mice: therapeutic relevance to anxiety disorders. Psychopharmacology 197: 601-611.

Liang KC, Juler RG, McGaugh JL (1986). Modulating effects of post-training epinephrine on memory: involvement of the amygdala noradrenergic system. Brain Res 368: 125-133.

Maren S, Quirk GJ (2004). Neuronal signalling of fear memory. Nat Rev Neurosci 5: 844-852.

McGaugh JL (1966). Time-dependent processes in memory storage. Science 153: 1351-1358.

McGaugh JL (2000). Memory - a century of consolidation. Science 287: 248-251.

McGaugh JL (2002). Memory consolidation and the amygdala: a systems perspective. Trends Neurosci 25: 456-461.

McGaugh JL, Roozendaal B (2009). Drug enhancement of memory consolidation: historical perspective and neurobiological implications. Psychopharmacology 202: 3-14.

Meis S, Bergado-Acosta JR, Yanagawa Y, Obata K, Stork O, Munsch T (2008). Identification of a neuropeptide $S$ responsive circuitry shaping amygdala activity via the endopiriform nucleus. PLoS One 3: e2695.

Moses SN, Cole C, Driscoll I, Ryan JD (2005). Differential contributions of hippocampus, amygdala and perirhinal cortex to recognition of novel objects, contextual stimuli and stimulus relationships. Brain Res Bull 67: 62-76.

National Research Council (2003). Guidelines for the Care and Use of Mammals in Neuroscience and Behavioral Research. National Academies Press: Washington, DC.

Nielson KA, Czech DA, Laubmeier KK (1999). Chronic administration of propranolol impairs inhibitory avoidance retention in mice. Neurobiol Learn Mem 71: 248-257.

Okamura N, Habay SA, Zeng J, Chamberlin AR, Reinscheid RK (2008). Synthesis and pharmacological in vitro and in vivo profile of 3-oxo-1,1-diphenyl-tetrahydro-oxazolo[3,4-a]pyrazine-7-carboxylic acid 4-fluoro-benzylamide (SHA 68), a selective antagonist of the neuropeptide $S$ receptor. J Pharmacol Exp Ther 325: 893-901.

Okamura N, Hashimoto K, Iyo M, Shimizu E, Dempfle A, Friedel S et al (2007). Gender-specific association of a functional coding polymorphism in the neuropeptide $S$ receptor gene with panic disorder but not with schizophrenia or attention-deficit/ hyperactivity disorder. Prog Neuropsychopharmacol Biol Psychiatry 31: $1444-1448$

Okamura N, Reinscheid RK (2007). Neuropeptide S: a novel modulator of stress and arousal. Stress 10: 221-226.

O'Mara S (2006). Controlling hippocampal output: the central role of subiculum in hippocampal information processing. Behav Brain Res 174: 304-312.

Pape HC, Paré D (2010). Plastic synaptic networks of the amygdala for the acquisition, expression, and extinction of conditioned fear. Physiol Rev 90: 419-463.

Patel JB, Ciofalo VB, Iorio LC (1979). Benzodiazepine blockade of passive-avoidance task in mice: a state-dependent phenomenon. Psychopharmacology 61: 25-28.

Phelps EA, LeDoux JE (2005). Contributions of the amygdala to emotion processing: from animal models to human behavior. Neuron 48: 175-187.

Quirarte GL, Roozendaal B, McGaugh JL (1997). Glucocorticoid enhancement of memory storage involves noradrenergic activation in the basolateral amygdala. Proc Natl Acad Sci USA 94: 14048-14053.

Reinscheid RK (2007). Phylogenetic appearance of neuropeptide $S$ precursor proteins in tetrapods. Peptides 28: 830-837.

Reinscheid RK, Xu YL, Okamura N, Zeng J, Chung S, Pai R et al (2005). Pharmacological characterization of human and murine neuropeptide S receptor variants. J Pharmacol Exp Ther 315: $1338-1345$.

Rizzi A, Vergura R, Marzola G, Ruzza C, Guerrini R, Salvadori S et al (2008). Neuropeptide $S$ is a stimulatory anxiolytic agent: a behavioural study in mice. Br J Pharmacol 154: 471-479.

Roozendaal B, Castello NA, Vedana G, Barsegyan A, McGaugh JL (2008). Noradrenergic activation of the basolateral amygdala modulates consolidation of object recognition memory. Neurobiol Learn Mem 90: 576-579.

Tang YP, Shimizu E, Dube GR, Rampon C, Kerchner GA, Zhuo M et al (1999). Genetic enhancement of learning and memory in mice. Nature 401: 63-69.

Winters BD, Forwood SE, Cowell RA, Saksida LM, Bussey TJ (2004). Double Dissociation between the effects of peripostrhinal cortex and hippocampal lesions on tests of object recognition and spatial memory: heterogeneity of function within the temporal lobe. J Neurosci 24: 5901-5908.

Xu YL, Gall CM, Jackson VR, Civelli O, Reinscheid RK (2007). Distribution of neuropeptide $S$ receptor mRNA and neurochemical characteristics of neuropeptide $S$-expressing neurons in the rat brain. J Comp Neurol 500: 84-102.

Xu YL, Reinscheid RK, Huitron-Resendiz S, Clark SD, Wang Z, Lin SH et al (2004). Neuropeptide S: a neuropeptide promoting arousal and anxiolytic-like effects. Neuron 43: 487-497.

Supplementary Information accompanies the paper on the Neuropsychopharmacology website (http://www.nature.com/npp) 